\title{
Molecular Magnetic Resonance Imaging with Contrast Agents for Assessment of Inflammatory Bowel Disease: A Systematic Review
}

\author{
Yifan Luo $\mathbb{D}^{1,2,3}$ Chen Gao $\left.\mathbb{D}^{1}\right)^{1,2}$ Wujie Chen $\mathbb{D}^{1,2}$ Kefeng Zhou $\mathbb{D}^{1,2}$ and Maosheng Xu $\mathbb{D}^{1,2}$ \\ ${ }^{1}$ The First Clinical Medical College of Zhejiang Chinese Medical University, Hangzhou 310053, China \\ ${ }^{2}$ Department of Radiology, The First Affiliated Hospital of Zhejiang Chinese Medical University, Hangzhou 310006, China \\ ${ }^{3}$ Department of Radiology, Tongde Hospital of Zhejiang Province, Hangzhou 310012, China
}

Correspondence should be addressed to Maosheng Xu; xums166@zcmu.edu.cn

Received 8 March 2020; Revised 20 April 2020; Accepted 25 April 2020; Published 6 May 2020

Academic Editor: Guillermina Ferro-Flores

Copyright (c) 2020 Yifan Luo et al. This is an open access article distributed under the Creative Commons Attribution License, which permits unrestricted use, distribution, and reproduction in any medium, provided the original work is properly cited.

Background and Aims. Magnetic resonance imaging (MRI) has taken an important role in the diagnosis of inflammatory bowel diseases (IBD). In the wake of current advances in nanotechnology, the drug delivery industry has seen a surge of nanoparticles advertising high specificity in target imaging. Given the rapid development of the field, this review has assembled related articles to explore whether molecular contrast agents can improve the diagnostic capability on gastrointestinal imaging, especially for IBD. Methods. Relevant articles published between 1998 and 2018 from a literature search of PubMed and EMBASE were reviewed. Data extraction was performed on the studies' characteristics, experimental animals, modelling methods, nanoparticles type, magnetic resonance methods, and means of quantitative analysis. Results. A total of 8 studies were identified wherein the subjects were animals, and all studies employed MR equipment. One group utilized a perfluorocarbon solution and the other 7 groups used either magnetic nanoparticles or gadolinium- (Gd-) related nanoparticles for molecular contrast. With ultrasmall superparamagnetic iron oxide (USPIO) particles and Gd-related nanoparticles, signal enhancements were found in the mucosa or with focal lesion of IBD-related model in T1-weighted images (T1WI), whereas superparamagnetic iron oxide (SPIO) particles showed a signal decrease in the intestinal wall of the model in T1WI or T2-weighted images. The signal-to-noise ratio (SNR) was employed to analyze bowel intensity in 3 studies. And the percentage of normalized enhancement was used in 1 study for assessing the severity of inflammation. Conclusion. Molecular MRI with contrast agents can improve the early diagnosis of IBD and quantitate the severity of inflammation in experimental studies.

\section{Introduction}

Inflammatory bowel disease (IBD), including Crohn's disease (CD) and ulcerative colitis (UC), is a chronic, relapsing illness. The worldwide incidence of IBD has increased over the years, especially in western countries and newly industrialized countries $[1,2]$.

As a method for noninvasive examination, radiology takes an important role in the diagnoses of IBD by evaluating lesions in the intestinal wall. Compared with computed tomography (CT), magnetic resonance imaging (MRI) obtains high-quality images without harmful ionizing radiation, making it a more suitable modality for patients who require long-term follow-up, teenagers, and young adults. With a higher spatial resolution as well as various sequences,
MRI is adapted to help diagnose IBD early and assess disease activity [3]. However, contrast-enhanced MRI sequences are often required for such diagnostic accuracy.

With the development of nanotechnology, new molecular probes have been developed for targeted imaging to evaluate lesions and surrounding tissues. The majority of the molecular contrast agents play a passive targeting role by being susceptible to macrophage clearance. Therefore, several studies rely on nanoparticles to monitor inflammation in the cardiovascular and nervous systems related to macrophage dysplasia [4-6]. On the other hand, active targeting probes can target track lesions via intracellular trapping. These modalities are mainly used in the evaluation of carcinomas and cardiovascular diseases [6]. It is worth noting that macrophage dysplasia also exists in intestinal 
inflammation and mucosal barrier damage in inflammatory regions increases permeability to some substances, providing a basis for the application of molecular imaging in the intestinal system $[7,8]$.

In this review, we assembled animal studies regarding magnetic resonance (MR) intestinal imaging using molecular contrast agents in the last twenty years. This review seeks to explore the advantages and characteristics of molecular imaging for the early diagnosis and quantitative assessment of IBD. We also assess the feasibility of molecular imaging for future clinical translation.

\section{Materials and Methods}

A systematic search of published studies in PubMed and EMBASE from 1998 to 2018 was conducted. Each database was searched for "magnetic resonance imaging" or "magnetic resonance" and "nanoparticle" or "nanoparticles" or "nanocrystalline material" or "nanocrystal" or "nanocrystals" and "gastrointestinal Tract" or "gastrointestinal tracts" or "GI tract" or "GI tracts" or "digestive tract" or "digestive tracts" or "digestive system". No language restrictions were applied, and the references from selected articles were also scanned for additional relevant studies. The protocol for this review was registered on PROSPERO (http://www.crd.york.ac.uk/PROSPERO;

CRD42019127236).

In the last two decades, molecular imaging was widely used to assess inflammation in the cardiovascular and nervous systems. However, literature regarding its use in intestinal imaging is scarce. Therefore, our group examined relevant literature without adding intestinal keywords to avoid excluding related articles.

Titles and abstracts of potentially relevant studies were examined to determine whether they fulfilled the following inclusion criteria (see Figure 1): (1) studies must apply molecular materials to intestinal imaging and (2) use MR equipment. Articles that explored intestinal imaging ex vivo, in vitro, and in silico models were excluded. After reading the full text, articles which used either a spontaneous intestinal tumor model or only used molecular materials as the neutral contrast agent were also excluded. In the end, all articles either (1) assessed molecular probes designed for IBD diagnosis using healthy animals or (2) assessed molecular imaging for IBD or colitis-associated colorectal cancer models.

Data extraction was performed for animal species, sex, weight, age, the type of molecular contrast agent, administration method, absorption mechanism, MR equipment, MR sequence, and histological confirmation.

\section{Results}

Our literature search yielded 848 potentially relevant studies which used nanoparticles in MRI studies. There were 840 studies excluded for reasons listed in Figure 1. Characteristics of the 8 included studies were summarized in Tables 1 and 2 .
3.1. Subjects. There were 8 studies matched the search criteria in which animal models of mice, rats, and rabbits were employed in articles 5, 2, and 1, respectively. Molecular MR imaging was facilitated in all 8 studies except for one, in which MRI was combined with single-photon emission computed tomography (SPECT/CT) [9]. Positive contrast agent was employed in 3 studies which showed abnormal strengthening in inflammation or colitis-associated dysplasia $[10,13,14]$. Another 2 groups used magnetic nanoparticles (MNPs) that showed signal intensity decreases in inflammatory lesions $[9,11]$. Actively targeted nanoparticles were used in 2 studies, which can reach the lower digestive tract through oral administration and improve intestinal imaging $[12,15]$. Perfluorocarbon (PFC) was adopted to tract macrophages in one study [16].

3.2. Experimental Animals. In the 5 murine studies, C57/B6, C56BL6, nude mice, and A/J mice were used, respectively $[9,11-13,16]$. The majority of studies used mice aged 5 to 8 weeks except for one study which used adult mice. Two studies adopted Lewis rats aged 8 to 12 weeks with an average weight of 220 to $270 \mathrm{~g}$ and Sprague Dawley rats with a 190 to $210 \mathrm{~g}$ body weight $[10,14]$. The final study used a healthy rabbit at $2.7 \mathrm{~kg}$ in mass [15]. For modelling methods, three studies used healthy animals $[12,14,15]$, two applied dextran sodium sulfate (DSS) to induce colitis in a mice model [9, 11], one used 2,4-dinitrobenzene sulfonic acid (DNBS) for colitis induction [10], and two groups treated mice with azoxymethane and DSS to generate a colitis-associated colorectal cancer model $[13,16]$.

3.3. Contrast Agents. PFC emulsions were used in 1 study [16] while other studies used nanoparticles. Four employed MNPs [9-12] and the other 3 adopted gadolinium- (Gd-) related nanoparticles [13-15].

3.3.1. MNPs. In the MNP group, studies used ferumoxides $\left(5 \times 10^{6}\right.$ superparamagnetic iron oxide (SPIO) nanoparticlelabeled macrophages per mice) [9], Nanotex $(0.5 \mathrm{mg} \mathrm{Fe} / \mathrm{ml}$, $100 \mu \mathrm{L}$ per mice) [11], and casein-coated indocyanine greenloaded iron oxide nanoparticles (CN-ICG-IO) $(10 \mathrm{mg} \mathrm{Fe} / \mathrm{kg})$ pertaining to SPIOs [12], while 1 study [10] used SHU 555C (5.6 mg Fe/kg) pertaining to USPIOs.

SHU 555C and Nanotex were taken up by the colon reticuloendothelial system (RES) to image inflammatory sites via intravenous administration [10, 11]. Wu et al.labelled macrophages with ferumoxide ex vivo and transfused the labelled cells back to the subject [9]. All studies located inflammatory sites by tracking the migration and distribution of macrophages.

In a separate study, CN-ICG-IO is encased by casein and modified with polyethylene glycol (PEG), which can be taken orally [12]. In another study, Nanotex combined with anti-inflammatory agents made it possible to integrate both diagnosis and treatment, creating a theranostic modality [11]. In histological confirmation, MNP groups used Prussian blue staining. 


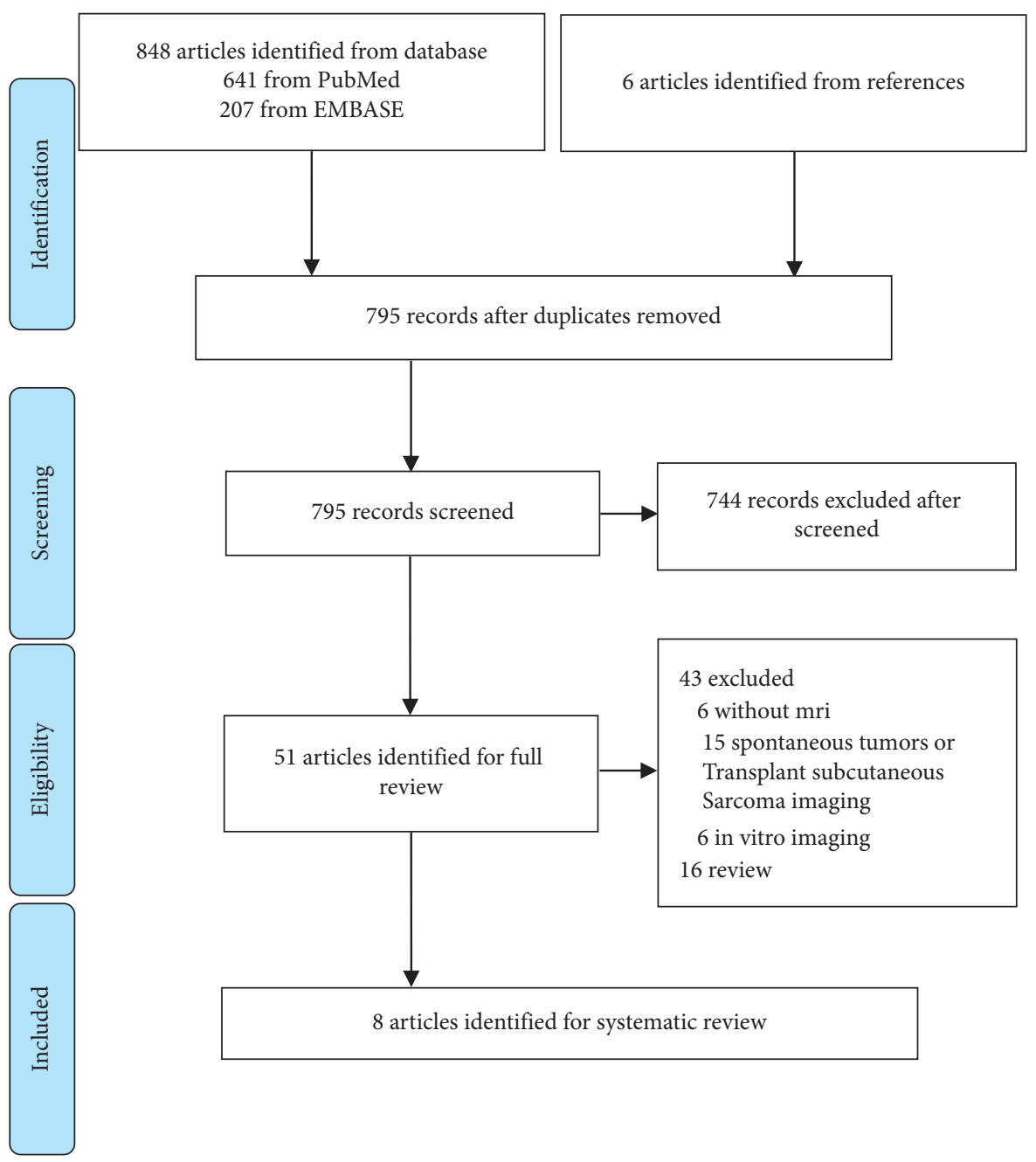

FIGURE 1: Flow diagram of the search strategy.

3.3.2. Gd-Related Nanoparticles. Sun et al. [13] treated animals with Gd-loaded solid lipid nanoparticles (SLNs) $(40 \mathrm{mg} / \mathrm{ml}, \mathrm{Gd}$-diethylenetriaminepentaacetic acid (GdDTPA) with a loading rate of $\sim 30 \%$ ) by enema. The nanoparticles showed preferential accumulation in lesion areas due to colitis-associated, high-grade intraepithelial neoplasia, which creates higher permeability than healthy tissue. Cheng et al. [14] used Gd-DTPA-loaded chitosan nanoparticles (Gd in chitosan) $(14.2 \mathrm{mg} / \mathrm{ml}$, Gd-DTPA with a loading rate of 74.4\%), and Perera et al. [15] used PEGcoated, Gd-incorporated, Prussian blue nanoparticles (Gd in PBNPs) (138.9 mg NPs $/ \mathrm{kg}$ ). Both of the aforementioned materials can be endocytosed to enhance colon mucosal uptake while the former is given by rectal administration and the latter is given by oral administration. In histological confirmation, Gd-loaded nanoparticles were combined with fluorescein isothiocyanate (FITC) to verify that material is absorbed by tissue [13].

3.3.3. Perfluorocarbon Solution. Shin et al. [16] treated colitis-associated dysplasia mice with PFC $(200 \mathrm{mg} / \mathrm{ml}, 200 \mu \mathrm{l}$ per mice) via tail vein iv injection. A ${ }^{19} \mathrm{~F}$ signal to noise was used to track labelled macrophages and assess the lesion area. In their study, inflammation in the descending colon was significantly increased compared to the ascending colon, while colitis-associated dysplasia and adenoma only appeared in descending colon. This finding proved that the occurrence of adenoma was closely related to that of inflammation.

3.4. MR Imaging Protocols. For the 5 murine studies, one group [11] used 7 T MRI equipment, one group [9] used 9.4 T MRI equipment, and one group [16] used 11.7 T MRI equipment while other groups $[12,13]$ used clinical $3.0 \mathrm{~T}$ or $3 \mathrm{~T}$ MRI equipment, and some studies $[11,16]$ mentioned the use of a $16-20 \mathrm{~mm}$ surface coil. In rat studies, one study [10] used a 2.4 T MRI and the other [14] used a clinical 3.0 T MRI equipment with a brain crossed coil. In the rabbit study, a clinical 1.5 T MRI was used [15].

For sequence selection, the PFC group [16] adopted a rapid acquisition with refocused echoes (RARE) sequence (repetition time (ms) (TR)/echo time (ms) $(\mathrm{TE})=1200 /$ $30 \mathrm{~ms}$ for ${ }^{1} \mathrm{H}$ images and TR/TE $=1000 / 14 \mathrm{~ms}$ for ${ }^{19} \mathrm{~F}$ images). In the MNP group, ferumoxides [9] used a T1- 
TABLE 1: A summary of molecular MR contrast agents.

\begin{tabular}{|c|c|c|c|c|c|c|}
\hline NPs & Material & Core & Coating & Animal model & Target & Characteristics \\
\hline \multirow{4}{*}{ MNPs } & Ferumoxides [9] & $\mathrm{Fe}_{3} \mathrm{O}_{4}$ & Dextran & $\begin{array}{l}\text { DSS-induced } \\
\text { colitis mice } \\
\text { model }\end{array}$ & $\begin{array}{c}\text { Macrophage (ex vivo cell } \\
\text { labelling) }\end{array}$ & Adept for cell tracking \\
\hline & SHU 555C [10] & $\begin{array}{l}\text { Iron } \\
\text { oxide }\end{array}$ & Carboxydextran & $\begin{array}{l}\text { DNBS-induced } \\
\text { colitis Lewis rat } \\
\text { model }\end{array}$ & RES & Positive contrast in MNPs \\
\hline & Nanotex [11] & $\mathrm{Fe}_{3} \mathrm{O}_{4}$ & SLN & $\begin{array}{l}\text { DSS-induced } \\
\text { colitis mice } \\
\text { model }\end{array}$ & RES & Drug loaded \\
\hline & CN-ICG-IO [12] & $\begin{array}{l}\text { Iron } \\
\text { oxide }\end{array}$ & Casein & $\begin{array}{l}\text { Healthy nude } \\
\text { mice }\end{array}$ & Gastrointestinal mucosal & Oral administration \\
\hline \multirow{3}{*}{$\begin{array}{l}\text { Gd- } \\
\text { related } \\
\text { NPs }\end{array}$} & Gd-FITC-SLN [13] & $\begin{array}{c}\text { Gd- } \\
\text { DTPA }\end{array}$ & SLN & $\begin{array}{l}\text { AOM- and DSS- } \\
\text { induced mice } \\
\text { model }\end{array}$ & $\begin{array}{c}\text { Colitis-associated } \\
\text { colorectal intraepithelial } \\
\text { neoplasia }\end{array}$ & $\begin{array}{l}\text { Absorbed via colon } \\
\text { mucosal membrane } \\
\text { functionality }\end{array}$ \\
\hline & $\begin{array}{l}\text { Gd in chitosan } \\
\text { nanoparticles [14] }\end{array}$ & $\begin{array}{l}\text { Gd- } \\
\text { DTPA }\end{array}$ & Chitosan & $\begin{array}{l}\text { Healthy Sprague } \\
\text { Dawley rats }\end{array}$ & Gastrointestinal mucosal & $\begin{array}{l}\text { Absorbed via colon } \\
\text { mucosal membrane } \\
\text { functionality }\end{array}$ \\
\hline & $\begin{array}{l}\text { PEG-coated Gd in } \\
\text { PBNPs [15] }\end{array}$ & $\begin{array}{l}\text { Gd in } \\
\text { PBNPs }\end{array}$ & PEG & Healthy rabbit & Gastrointestinal mucosal & $\begin{array}{c}\text { Oral administration, PBNP } \\
\text { increases absorptivity }\end{array}$ \\
\hline PFC & PFC emulsion [16] & - & - & $\begin{array}{l}\text { AOM- and DSS- } \\
\text { induced mice } \\
\text { model }\end{array}$ & Macrophage & $\begin{array}{l}\text { Metal ions free, adept for } \\
\text { cell tracking }\end{array}$ \\
\hline
\end{tabular}

AOM, azoxymethane; CN-ICG-IO, casein-coated indocyanine green loaded iron oxide nanoparticle; DNBS, 2,4-dinitrobenzene sulfonic acid; DSS, dextran sodium sulfate; DTPA, diethylenetriaminepentaacetic acid; FITC, fluorescein isothiocyanate; Gd, gadolinium; MNPs, magnetic nanoparticles; NPs, nanoparticles; PBNPs, Prussian blue nanoparticles; PEG, polyethylene glycol; PFC, perfluorocarbon; RES, reticuloendothelial system; SLN, solid lipid nanoparticles.

TABLE 2: The applications of molecular contrast agents MRI in IBD-related model.

\begin{tabular}{|c|c|c|c|c|c|c|}
\hline NPs & Material & $\begin{array}{c}\text { Field } \\
\text { strength }\end{array}$ & Sequences & Enhancement ${ }^{\mathrm{a}}$ & Administration & Procedures \\
\hline \multirow{4}{*}{ MNPs } & Ferumoxides [9] & $9.4 \mathrm{~T}$ & T1WI & Intestinal wall T1WI $(\downarrow)$ & iv & $\begin{array}{c}\text { Long enhancement sustained time, } \\
\text { quantitative analysis }\end{array}$ \\
\hline & SHU 555C [10] & $2.4 \mathrm{~T}$ & $\begin{array}{l}\text { T1WI, } \\
\text { T2WI, T2* }\end{array}$ & $\begin{array}{c}\text { Mucosa T1WI }(\uparrow) \text {, deep } \\
\text { colon wall T1WI }(\downarrow) \\
\text { \&T2WI }(\downarrow)\end{array}$ & iv & Quantitative analysis \\
\hline & Nanotex [11] & $7 \mathrm{~T}$ & T1WI & Colon wall T1WI $(\downarrow)$ & iv & $\begin{array}{c}\text { Integration of diagnosis and } \\
\text { treatment }\end{array}$ \\
\hline & CN-ICG-IO [12] & $3 \mathrm{~T}$ & T2WI & $\mathrm{NA}^{\mathrm{b}}$ & po & $\begin{array}{l}\text { Oral administration, potential to } \\
\text { monitor inflammation and targeted } \\
\text { delivery }\end{array}$ \\
\hline \multirow{3}{*}{$\begin{array}{l}\text { Gd- } \\
\text { related } \\
\text { NPs }\end{array}$} & Gd-FITC-SLN [13] & $3.0 \mathrm{~T}$ & T1WI & $\begin{array}{l}\text { Focal lesion } \\
\text { T1WI }(\uparrow)\end{array}$ & pr & $\begin{array}{l}\text { Targeted imaging through intestinal } \\
\text { absorption, low Gd deposition, } \\
\text { quickly metabolized }\end{array}$ \\
\hline & $\begin{array}{l}\text { Gd in chitosan } \\
\text { nanoparticles }[14]\end{array}$ & $3.0 \mathrm{~T}$ & $\mathrm{~T} 1 \mathrm{~W}$ & Mucosa T1WI $(\uparrow)^{\mathrm{b}}$ & pr & $\begin{array}{l}\text { Targeted imaging through intestinal } \\
\text { absorption, low Gd deposition }\end{array}$ \\
\hline & $\begin{array}{l}\text { PEG-coated Gd in } \\
\text { PBNPs [15] }\end{array}$ & $1.5 \mathrm{~T}$ & T1WI & $\mathrm{NA}^{\mathrm{b}}$ & po & $\begin{array}{c}\text { Potential to monitor inflammation } \\
\text { or targeted delivery, low Gd } \\
\text { deposition, oral administration }\end{array}$ \\
\hline PFC & PFC emulsion [16] & $11.7 \mathrm{~T}$ & T1WI & $\begin{array}{c}\text { Special }{ }^{19} \mathrm{~F} \text { signal in focal } \\
\text { lesion }\end{array}$ & iv & $\begin{array}{l}\text { Quantitative analysis; not affected } \\
\text { by bowel movements or the } \\
\text { existence of air pocket }\end{array}$ \\
\hline
\end{tabular}

CN-ICG-IO, casein-coated indocyanine green-loaded iron oxide nanoparticle; FITC, fluorescein isothiocyanate; Gd, gadolinium; iv, intravenously; MNPs, magnetic nanoparticles; NA, not available; NPs, nanoparticles; PBNPs, Prussian blue nanoparticles; PEG, polyethylene glycol; PFC, perfluorocarbon; po, per os; pr, per rectum; T1WI, T1-weighted image; T2WI, T2-weighted image. Notes: ${ }^{\mathrm{a}} \mathrm{MNPs}$ have both T1W and T2W effect, and these show the major effect with suitable dose in corresponding experiments; bhealthy animals were used in the experiment; $(\uparrow)$ signal increased; $(\downarrow)$ signal decreased. 
weighted (T1W) black-blood spin-echo sequence (TR/TE, $600 / 8.6 \mathrm{~ms}$ ), Nanotex [11] employed a T1W images by using a multislice multiecho sequence $(\mathrm{TR} / \mathrm{TE}=521 / 10.6 \mathrm{~ms}), \mathrm{CN}$ ICG-IO [12] used a T2-weighted (T2W) fast spin-echo sequence $(\mathrm{TR} / \mathrm{TE}=3600 / 86 \mathrm{~ms})$, and SHU 555C [10] used a T1W 2D spin-echo sequence $(\mathrm{TR} / \mathrm{TE}=680 / 12.3 \mathrm{~ms}), \mathrm{T} 2 \mathrm{~W}$ $2 \mathrm{D}$ turbo-spin-echo sequence $(\mathrm{TR} / \mathrm{TE}=3300 / 77.2 \mathrm{~ms})$, and a T2*-weighted 2D gradient-recalled echo sequence (TR/ $\mathrm{TE}=905 / 25.4 \mathrm{~ms}$ ). For Gd-related nanoparticles, Gd-FITCSLN [13] used a T1W spin-echo sequence $(\mathrm{TR} / \mathrm{TE}=550$ / $15 \mathrm{~ms}$ ) and Gd in chitosan [14] nanoparticles used a T1W spectral presaturation inversion recovery sequence (TR/ $\mathrm{TE}=456.47 / 14.5 \mathrm{~ms}$ ) and T1W high-resolution isotropic volume examination sequence $(\mathrm{TR} / \mathrm{TE}=7.97 / 3.94 \mathrm{~ms})$ was used for the for Gd-DTPA control group. Finally, PEGcoated Gd in PBNPs [15] used the 3D FLASH sequence.

\subsection{Molecular Imaging and Improved Diagnostics.} Gd-related contrast agents [13, 14] allowed significant enhancement in image quality for T1WI. Gd-based nanoparticles $[13,14]$ administered rectally showed a substantial increase in signal intensity along with the control group of Gd-DTPA. Nanoparticle-based gadolinium administered orally showed signal increases as well [15], through the addition of Prussian blue, and the aforementioned modality achieved notable increases in signal through endocytosis by intestinal epithelial cells.

Mucosal enhancement was observed in T1WI of rats with colitis after USPIO administration [10]. Meanwhile, deeper colon wall structures showed hyperintensity on unenhanced T2WI which presented as hypointensities after contrast enhancement. Two groups treated DSSinduced colitis with MNPs via intravenous injection $[9,11]$; it was found that signal intensity decreased in T1WI. However, due to inherent artifacts or susceptibility artifacts, gradient-echo sequences, and $\mathrm{T}_{2}{ }^{*}$, these sequences could not be used $[9,10]$. An additional study demonstrated severe signal distortion through excessive material concentration [12].

The PFC group employed ParaVision software to combine a pseudocolor ${ }^{19} \mathrm{~F}$ signal with ${ }^{1} \mathrm{H}$ imaging [16]. This allowed the tracking and assessment of intestinal inflammation through the distribution of the ${ }^{19} \mathrm{~F}$ signal.

Finally, three groups utilized the signal-to-noise ratio (SNR) to analyze the region of interest $[10,13,16]$. The SNR was calculated by dividing the signal intensity of the samples by the standard deviation of the background noise [17]. Finally, one group utilized the percentage normalized enhancement $(\% \mathrm{NENH})$ between the intestine and liver for quantitative evaluation [16].

\section{Discussion}

4.1. Molecular Contrast Agent Selection. MNPs are nanoparticles with iron and iron oxides as the main magnetic substances which have a higher relaxation degree and stronger effect on local magnetic fields than gadolinium-type molecular probes [18]. These include superparamagnetic iron oxide (SPIO) and ultrasmall superparamagnetic iron oxide (USPIO) particles. SPIOs such as ferucarbotran (SHU $555 \mathrm{~A}$ ) are often more than $20 \mathrm{~nm}$ in diameter, with a core of $\mathrm{Fe}_{3} \mathrm{O}_{4}$ or $\mathrm{Fe}_{2} \mathrm{O}_{3}$ and modification with carbon, oxygen, or glucose. Due to the diameter of SPIO particles, they suffer from reticuloendothelial uptake in the liver and spleen. Meanwhile, the diameter of USPIOs is less than $20 \mathrm{~nm}$, with an iron oxide core and dextran on the surface. Their small size permits them to enter lymphatic circulation and bone marrow $[18,19]$.

According to different chemical compositions, particle sizes, and surface properties, MNPs can be divided into T1W agents and T2W agents [20]. SPIO classifies as a T2W agent while USPIO has a greater T1 effect. The characteristics of positive enhancement make USPIO more suitable for diagnostic use. Like other organs and tissues, the MNP system has the ability to enter the colonic RES through circulation $[9,10]$. Yet, signal distortion can negatively impact diagnosis if the local concentration is too high $[10,12]$.

Compared with the MNPs, gadolinium-like molecular probes do not have magnetic susceptibility artifacts. SLNs are a type of colloidal particle made from protein, dendrimer, or liposomes. Their sizes range between 50 and $1000 \mathrm{~nm}$ and they are biocompatible, biodegradable, and easily absorbed by the digestive tract [21].

Leukocytes, such as macrophages, are commonly recruited in inflammatory reactions. Because of this, their migration can be traced using a molecular contrast agent (Figure 2). While most contrast agents require intravenous administration, SLNs are mainly absorbed via the intestinal tract (Figure 3). When given orally, absorption by the stomach accounts for less than $5 \%$ of total uptake [29]. It has been reported that about 50\% of SLNs can be absorbed directly through intestinal mucosa or intercellularly, and the absorption correlates linearly within a certain concentration [21]. Specifically, M-cells overlying the lymphoid follicles and Peyer's patches may be the main ways SLNs enter the gut mucosa [25]. About $70 \%$ of absorbed SLNs were taken in circulation through the lymphatic system [21]. The remainder was transported directly through blood circulation, possibly via capillaries or intestinal epithelial cells by the paracellular route [26-28].

However, different conditions exist in pathological situations. Wu et al. [30] revealed that in DMH-treated mice, SLNs were primarily absorbed via carcinogenic colorectal mucosa and directly into the submucosal capillary network. Other studies have shown that the cell junction protein, zonula occludens 1 , was imparted in the inflammatory state [8]. This led to expansions in the cell junctions and increased permeability, resulting in increased uptake in the carcinogenic regions. Using lymphatic transport, SLNs were able to avoid first past metabolism in the liver and increase absorption and efficacy.

Some controversy exists regarding the absorption of SLNs through the gut. One study demonstrated that detection of the SLN complex in intestinal epithelial cells was difficult when using a water-quenching, near-infrared (NIR) fluorescent probe [31]. Another experiment showed that 


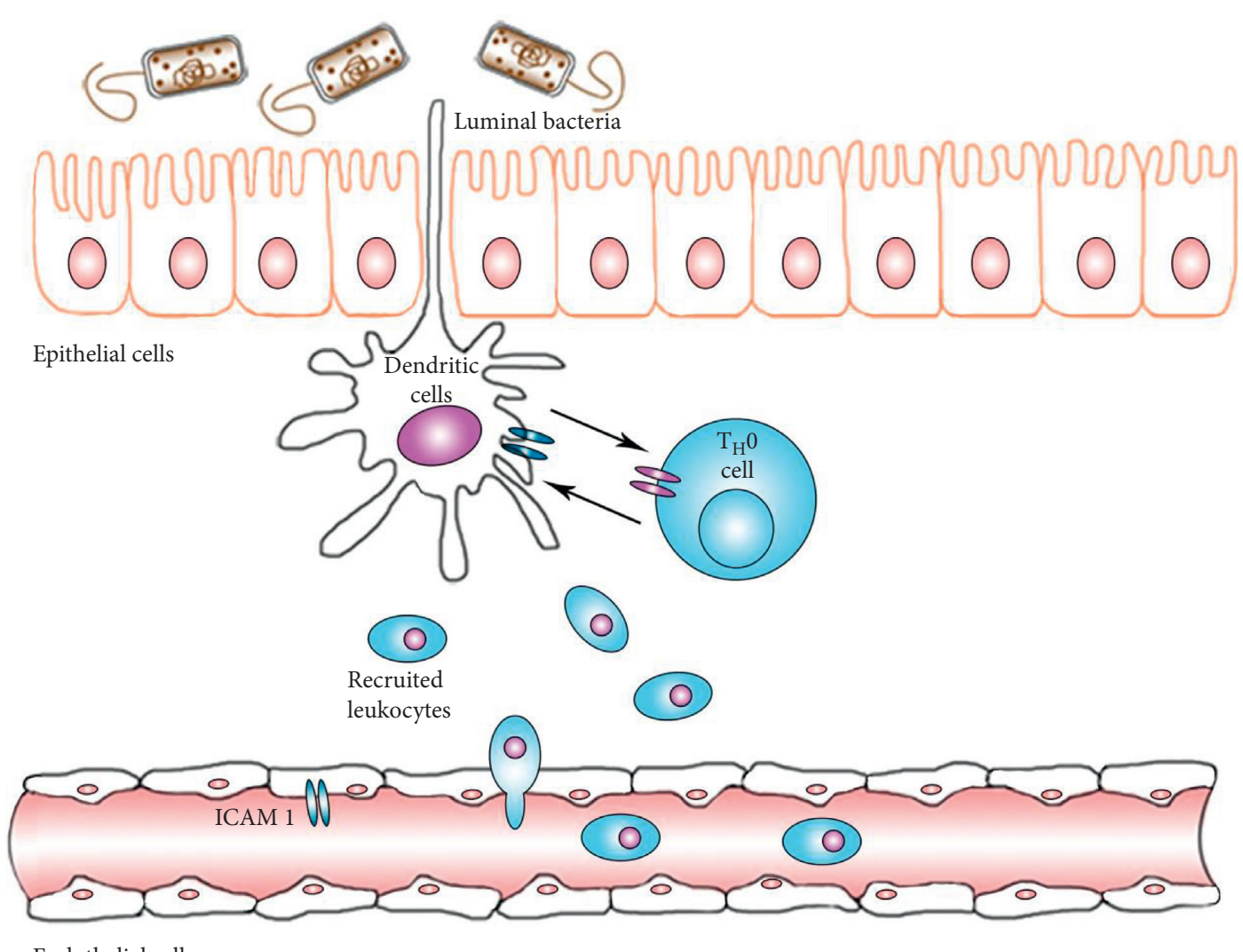

Endothelial cells

FIGURE 2: Leukocytes are recruited in the interstitial inflammatory response. Changes in the intestinal flora or a defective microbial clearing process may induce uncontrolled microbial growth and activation of proinflammatory processes [22]. There exists a mutual effect between dendritic cells (antigen-presenting cells) and naive T cells $\left(\mathrm{T}_{\mathrm{H}} 0\right)$. Following activation, activated cells will differentiate and release various cytokines. Stimulated intestinal epithelial cells can secrete ICAM 1, which will recruit monocytes such as mononuclear cells, polymorphonuclear cells, and lymphocytes [7, 23, 24].

SLNs with loaded drugs can pass through epithelial cells into intestinal capillaries, while lipids remain mostly in cells [32]. The characteristics of SLNs may be affected by different surface modifications as well as different manufacturing processes. Yet, multiple animal experiments have confirmed that SLNs can greatly improve the uptake and transport of the drugs loaded by intestinal epithelial cells $[25,33,34]$.

For nanoparticle surface modifications, Gd in PBNPs utilized PEG to improve the sustained release and extend the therapeutic window [15]. Both animal experiments [35] and clinical studies [36] found that PEG-modified drugs were more inclined to deposit at the lesion, which may be related to the abnormal state of intestinal mucosa during inflammation. Additionally, it was found that Prussian blue can also be used in conjunction to enhance the drugs' penetration [20].

The use of ${ }^{19} \mathrm{~F}$ to trace cell migration in MRI imaging is based on the sensitivity of the ${ }^{19} \mathrm{~F}$ nucleus relative to ${ }^{1} \mathrm{H}$. Compared to metal ion contrast agents, PFC is nontoxic and has been applied for cell tracking in a clinical setting [37].

For the use of contrast agents, $\mathrm{Wu}$ et al. [9] reinfused labelled macrophages via tail vein intravenously. Three studies $[10,11,16]$ adopted intravenous injection, two studies $[13,14]$ used rectal administration, and the other two studies [12, 15] used oral ingestion. Aiming to improve intestinal absorption, the nonintravenous groups used SLNs, chitosan, casein, or PEG to wrap the core material. Except for chitosan-based NPs, all other materials allow for oral administration. Compared to intravenous administration, the oral route is far less invasive with fewer side effects and improved compliance.

4.2. Imaging Optimization. For the development of the animal study, three key modalities were used. DSS induces inflammation in intestinal indiscriminately, while 2, 4, 6trinitrobenzene sulfonic acid (TNBS) and DNBS can induce partial colorectal inflammation through topical administration. Therefore, TNBS and DNBS may provide an opportunity to observe the difference between normal tissue and inflamed intestinal tissue in the same segment.

The majority of studies used mice aged 5-8 weeks, which provided a better model for bowel inflammation. Acute models are suitable for analyzing short-term barrier changes and innate immune effects and flares. On the other hand, chronic models are able to illustrate the role of adaptive immunity, neoplasia, and tissue fibrosis [38]. While a rat or rabbit model has advantages for imaging due to their size, murine models can also 


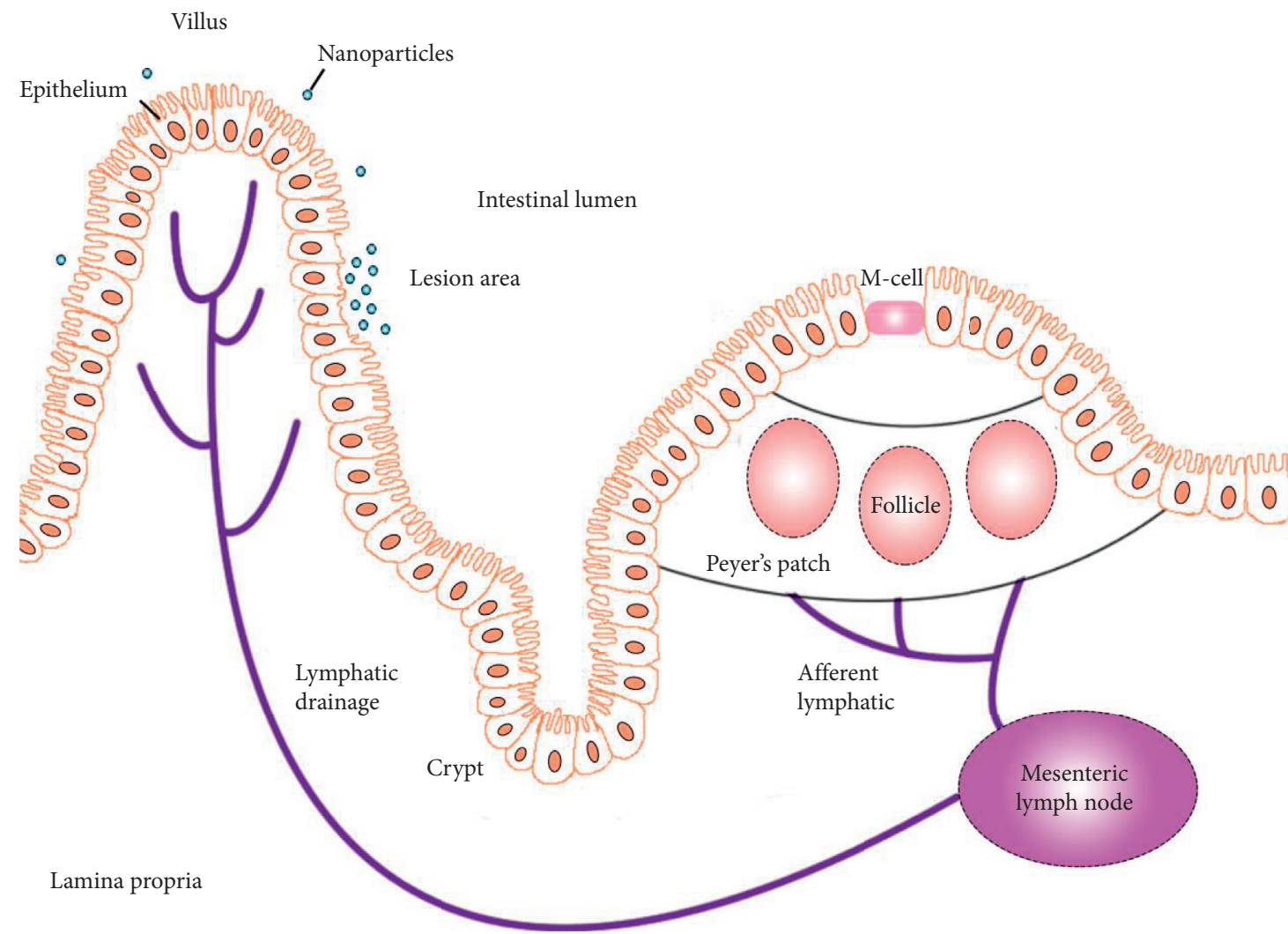

FIGURE 3: The absorption pathway of SLN. Lymphatic circulation plays an important role in SLN absorption [25]. Typically, most SLNs are either taken up by the M-cells overlying the lymphoid follicles, Peyer's patches, and circumambient lymphatic drainage, or transported via capillaries in direct or paracellular pathways [26-28]. However, in pathological situations, SLNs are primarily absorbed into the submucosal capillary network through the impaired colorectal mucosa. Furthermore, PEG-modified nanoparticles were more inclined to deposit at this lesion.

achieve the same resolution if an appropriate coil is used to display the intestinal wall contour $[13,30]$.

Intestinal tract peristalsis and pneumatosis can induce nonspecific hypointensities and complicate the use of SPIO for the diagnosis of bowel diseases. To combat this issue, $\mathrm{Wu}$ et al. [9] treated mice with $\mathrm{MnCl}_{2}$ to delineate the intestinal contour, which allowed SPIO to distinguish the bowel wall from the intestinal cavity. Currently, oral administration of $\mathrm{MnCl}_{2}$ is approved by the Food and Drug Administration [39].

With gadolinium-like molecular probes, it has been reported that fluid-attenuated inversion recovery (FLAIR) is more sensitive to low-concentration gadolinium imaging [40]. In light of this, one study used FLAIR to collected highresolution images in mice on a $1.5 \mathrm{~T}$ clinical scanner [30].

4.3. Future Expectations. Nanomaterials allow an unparalleled versatility in their ability to be loaded with therapeutic agents, genetic material, and various other compounds for treatment. For instance, Ohno et al. [41] treated murine colitis with nanoparticles loaded with curcumin. Zhang et al. [42] synthesized a redox-responsive b-cyclodextrin (OxbCD) and prepared Tempol- (TpL-) loaded OxbCD particles (TpL/OxbCD NPs) for the treatment of colitis in a murine model using oral administration. Separately, Huang et al. [33] prepared nucleic acid-loaded nanoparticles targeted to colonic macrophages. When given orally, these nanoparticles can be loaded with therapeutic agents that could be applied clinically. However, their studies were unable to monitor the activity level or drug distribution of intestinal inflammation in vivo.

Nanoparticles can also possess multiple functions when used as theranostic agents. Theranostic agents are agents which combine diagnosis and treatment, such as the combination of a contrast agent with a drug. Several studies have reported this method and found success in imaging using an in vivo imaging system or MRI $[34,43]$.

Despite the advantages of nanomedicine-based treatments, the major side effects primarily derive from the drug loaded within the carrier. For instance, recent studies have shown that gadolinium may exhibit deposition in the brain [44]. While concerning, no neurological symptoms have been reported to date [45]. Yet, gadolinium is contraindicated in patients with renal dysfunction due to the increased risk of nephrogenic fibrosis [44]. Finally, oral administration or enema administration has been shown to decrease side effects compared with iv injection, indicating the important role of the route of administration.

In spite of the findings listed herein, some limitations to the current study still exist. Firstly, only a few cases were recruited in each zoopery. Secondly, all research studies conducted were with animal studies, and further exploration 
is required to highlight the advantages that molecular MRI can have on the diagnosis of IBD for clinical trials. Thirdly, the sensitivity of MRI molecular imaging (micromolar) in comparison with positron emission tomography (picomolar) has a gap [46]. Through advances in molecular biology, nanoparticle-based targeted carriers, and bioengineering, the sensitivity of MRI for molecular imaging has risen. Undoubtedly, MRI imaging has proven itself as a highly versatile modality for the molecular imaging of inflammatory bowel disease, particularly for nanoscience-based delivery approaches.

\section{Conclusions}

With the development of a nanoparticle-based MR contrast agent, molecular MR imaging of IBD may improve and allow for (1) a comprehensive visual image of complex biological processes, demonstrating a new way to understand the molecular mechanisms of IBD; (2) monitoring of multiple processes at the same time; (3) improvement of early detection of IBD or metaplasia; and (4) monitoring of the mechanistic of drug delivery and efficacy in vivo. Furthermore, the advent of theranostics has been paving the way for combined modalities incorporating both diagnostics and therapy. When used in conjunction with MRI, nanomedicine-based therapies can greatly improve the diagnosis and treatment of IBD. The absorption characteristics of the intestinal tract provide a convenient basis for the oral administration of nanoparticles, which both improves patient compliance and decreases the incidence of adverse effects.

\section{Conflicts of Interest}

The authors declare that there are no conflicts of interest regarding the publication of this paper.

\section{Acknowledgments}

The authors thank Professor Jihong Sun from Sir Run Run Shaw Hospital for useful discussions during the progression of the work. This work was supported by the National Natural Science Foundation of China (81971600) and Natural Science Foundation of Zhejiang Province of China (LSY19H030001 and LSY19H180003).

\section{References}

[1] N. A. Molodecky, I. S. Soon, D. M. Rabi et al., "Increasing incidence and prevalence of the inflammatory bowel diseases with time, based on systematic review," Gastroenterology, vol. 142, no. 1, pp. 46.e42-54.e42, 2012.

[2] G. G. Kaplan, "The global burden of IBD: from 2015 to 2025," Nature Reviews Gastroenterology \& Hepatology, vol. 12, no. 12, pp. 720-727, 2015.

[3] H. Jadvar and P. M. Colletti, "Competitive advantage of PET/ MRI," European Journal of Radiology, vol. 83, no. 1, pp. 84-94, 2014.

[4] V. Amirbekian, M. J. Lipinski, K. C. Briley-Saebo et al., "Detecting and assessing macrophagesin vivoto evaluate atherosclerosis noninvasively using molecular MRI,"
Proceedings of the National Academy of Sciences, vol. 104, no. 3, pp. 961-966, 2007.

[5] R. A. Trivedi, C. Mallawarachi, J.-M. U-King-Im et al., "Identifying inflamed carotid plaques using in vivo USPIOenhanced MR imaging to label plaque macrophages," Arteriosclerosis, Thrombosis, and Vascular Biology, vol. 26, no. 7, pp. 1601-1606, 2006.

[6] P. Padmanabhan, A. Kumar, S. Kumar, R. K. Chaudhary, and B. Gulyás, "Nanoparticles in practice for molecular-imaging applications: an overview," Acta Biomaterialia, vol. 41, pp. 1-16, 2016.

[7] G. Y. Melmed and S. R. Targan, "Future biologic targets for IBD: potentials and pitfalls," Nature Reviews Gastroenterology \& Hepatology, vol. 7, no. 2, pp. 110-117, 2010.

[8] J. R. Turner, M. M. Buschmann, I. Romero-Calvo, A. Sailer, and L. Shen, "The role of molecular remodeling in differential regulation of tight junction permeability," Seminars in Cell \& Developmental Biology, vol. 36, pp. 204-212, 2014.

[9] Y. Wu, K. Briley-Saebo, J. Xie et al., "Inflammatory bowel disease: MR- and SPECT/CT-based macrophage imaging for monitoring and evaluating disease activity in experimental mouse model-pilot study," Radiology, vol. 271, no. 2, pp. 400-407, 2014.

[10] B. B. Frericks, F. Wacker, C. Loddenkemper et al., "Magnetic resonance imaging of experimental inflammatory bowel disease," Investigative Radiology, vol. 44, no. 1, pp. 23-30, 2009.

[11] D. Calle, V. Negri, P. Ballesteros, and S. Cerdán, "Magnetoliposomes loaded with poly-unsaturated fatty acids as novel theranostic anti-inflammatory formulations," Theranostics, vol. 5, no. 5, pp. 489-503, 2015.

[12] J. Huang, Q. Shu, L. Wang, H. Wu, A. Y. Wang, and H. Mao, "Layer-by-layer assembled milk protein coated magnetic nanoparticle enabled oral drug delivery with high stability in stomach and enzyme-responsive release in small intestine," Biomaterials, vol. 39, pp. 105-113, 2015.

[13] J. Sun, S. Zhang, S. Jiang et al., "Gadolinium-loaded solid lipid nanoparticles as a tumor-absorbable contrast agent for early diagnosis of colorectal tumors using magnetic resonance colonography," Journal of Biomedical Nanotechnology, vol. 12, no. 9, pp. 1709-1723, 2016.

[14] J.-J. Cheng, J. Zhu, X.-S. Liu et al., "Gadolinium-chitosan nanoparticles as a novel contrast agent for potential use in clinical bowel-targeted MRI: a feasibility study in healthy rats," Acta Radiologica, vol. 53, no. 8, pp. 900-907, 2012.

[15] V.S. Perera, G. Chen, Q. Cai, and S. D. Huang, "Nanoparticles of gadolinium-incorporated Prussian blue with PEG coating as an effective oral MRI contrast agent for gastrointestinal tract imaging," The Analyst, vol. 141, no. 6, pp. 2016-2022, 2016.

[16] S. H. Shin, D. K. Kadayakkara, and J. W. M Bulte, "In vivo (19) F MR imaging cell tracking of inflammatory macrophages and site-specific development of colitis-associated dysplasia," Radiology, vol. 282, no. 1, pp. 194-201, 2016.

[17] Y. Meng, J. Wang, J. Zhang et al., "3.0-T MR imaging of intracoronary local delivery of motexafin gadolinium into coronary artery walls," Radiology, vol. 268, no. 2, pp. 556-562, 2013.

[18] H. Ittrich, K. Peldschus, N. Raabe, M. Kaul, and G. Adam, "Superparamagnetic iron oxide nanoparticles in biomedicine: applications and developments in diagnostics and therapy," RöFo-Fortschritte auf dem Gebiet der Röntgenstrahlen und der bildgebenden Verfahren, vol. 185, no. 12, pp. 1149-1166, 2013. 
[19] M. F. Casula, P. Floris, C. Innocenti et al., "Magnetic resonance imaging contrast agents based on iron oxide superparamagnetic ferrofluids," Chemistry of Materials, vol. 22, no. 5, pp. 1739-1748, 2010.

[20] M. Shokouhimehr, E. S. Soehnlen, J. Hao et al., "Dual purpose Prussian blue nanoparticles for cellular imaging and drug delivery: a new generation of T1-weighted MRI contrast and small molecule delivery agents," Journal of Materials Chemistry, vol. 20, no. 25, p. 5251, 2010.

[21] H. Yuan, J. Chen, Y.-Z. Du, F.-Q. Hu, S. Zeng, and H.-L. Zhao, "Studies on oral absorption of stearic acid SLN by a novel fluorometric method," Colloids and Surfaces B: Biointerfaces, vol. 58, no. 2, pp. 157-164, 2007.

[22] S. E. M. Heinsbroek and S. Gordon, "The role of macrophages in inflammatory bowel diseases," Expert Reviews in Molecular Medicine, vol. 11, p. e14, 2009.

[23] D. Haller, M. P. Russo, R. B. Sartor, and C. Jobin, "IKK $\beta$ and phosphatidylinositol 3-kinase/akt participate in non-pathogenic gram-negative enteric bacteria-induced RelA phosphorylation and NF- $\kappa$ B activation in both primary and intestinal epithelial cell lines," Journal of Biological Chemistry, vol. 277, no. 41, pp. 38168-38178, 2002.

[24] R. B. Sartor, "Mechanisms of Disease: pathogenesis of Crohn's disease and ulcerative colitis," Nature Clinical Practice Gastroenterology \& Hepatology, vol. 3, no. 7, pp. 390-407, 2006.

[25] A. M. Mowat, "Anatomical basis of tolerance and immunity to intestinal antigens," Nature Reviews Immunology, vol. 3, no. 4, pp. 331-341, 2003.

[26] D.-D. Kim, H.-J. Cho, J. W. Park, and I.-S. Yoon, "Surfacemodified solid lipid nanoparticles for oral delivery of docetaxel: enhanced intestinal absorption and lymphatic uptake," International Journal of Nanomedicine, vol. 9, pp. 495-504, 2014.

[27] R. J. Bennink, C. van Montfrans, W. J. de Jonge, K. de Bruin, S. J. van Deventer, and A. A. te Velde, "Imaging of intestinal lymphocyte homing by means of pinhole SPECT in a TNBS colitis mouse model," Nuclear Medicine and Biology, vol. 31, no. 1, pp. 93-101, 2004.

[28] C. Charpentier, R. Marion-Letellier, G. Savoye et al., "Magnetic resonance colonography in rats with TNBS-induced colitis: a feasibility and validation study," Inflammatory Bowel Diseases, vol. 18, no. 10, pp. 1940-1949, 2012.

[29] Z. Zhang, F. Gao, H. Bu, J. Xiao, and Y. Li, "Solid lipid nanoparticles loading candesartan cilexetil enhance oral bioavailability: in vitro characteristics and absorption mechanism in rats," Nanomedicine: Nanotechnology, Biology and Medicine, vol. 8, no. 5, pp. 740-747, 2012.

[30] T. Wu, W.-L. Zheng, S.-Z. Zhang et al., "Bimodal visualization of colorectal uptake of nanoparticles in dimethylhydrazinetreated mice," World Journal of Gastroenterology, vol. 17, no. 31, pp. 3614-3622, 2011.

[31] X. Hu, W. Fan, Z. Yu et al., "Evidence does not support absorption of intact solid lipid nanoparticles via oral delivery," Nanoscale, vol. 8, no. 13, pp. 7024-7035, 2016.

[32] C. Chen, X. Zhu, Y. Dou et al., "Exendin-4 loaded nanoparticles with a lipid shell and aqueous core containing micelles for enhanced intestinal absorption," Journal of Biomedical Nanotechnology, vol. 11, no. 5, pp. 865-876, 2015.

[33] Z. Huang, J. Gan, L. Jia et al., "An orally administrated nucleotide-delivery vehicle targeting colonic macrophages for the treatment of inflammatory bowel disease," Biomaterials, vol. 48, pp. 26-36, 2015.

[34] M. Zhang, C. Xu, D. Liu, M. K. Han, L. Wang, and D. Merlin, "Oral delivery of nanoparticles loaded with ginger active compound, 6-shogaol, attenuates ulcerative colitis and promotes wound healing in a murine model of ulcerative colitis," Journal of Crohn's and Colitis, vol. 12, no. 2, pp. 217-229, 2018.

[35] K. Maisel, L. Ensign, M. Reddy, R. Cone, and J. Hanes, "Effect of surface chemistry on nanoparticle interaction with gastrointestinal mucus and distribution in the gastrointestinal tract following oral and rectal administration in the mouse," Journal of Controlled Release, vol. 197, pp. 48-57, 2015.

[36] C. Lautenschläger, C. Schmidt, C.-M. Lehr, D. Fischer, and A. Stallmach, "PEG-functionalized microparticles selectively target inflamed mucosa in inflammatory bowel disease," European Journal of Pharmaceutics and Biopharmaceutics, vol. 85, no. 3, pp. 578-586, 2013.

[37] E. T. Ahrens, B. M. Helfer, C. F. O’Hanlon, and C. Schirda, "Clinical cell therapy imaging using a perfluorocarbon tracer and fluorine-19 MRI," Magnetic Resonance in Medicine, vol. 72, no. 6, pp. 1696-1701, 2014.

[38] S. Wirtz, V. Popp, M. Kindermann et al., "Chemically induced mouse models of acute and chronic intestinal inflammation," Nature Protocols, vol. 12, no. 7, pp. 1295-1309, 2017.

[39] P. Leander, K. Golman, S. Månsson, and P. Höglund, "Orally administered manganese with and without ascorbic acid as a liver-specific contrast agent and bowel marker for magnetic resonance imaging," Investigative Radiology, vol. 45, no. 9, pp. 559-564, 2010.

[40] N. Ercan, S. Gultekin, H. Celik, T. E. Tali, Y. A. Oner, and G. Erbas, "Diagnostic value of contrast-enhanced fluid-attenuated inversion recovery MR imaging of intracranial metastases," American Journal of Neuroradiology, vol. 25, no. 5, pp. 761-765, 2004.

[41] M. Ohno, A. Nishida, Y. Sugitani et al., "Nanoparticle curcumin ameliorates experimental colitis via modulation of gut microbiota and induction of regulatory T cells," PLoS One, vol. 12, no. 10, Article ID e0185999, 2017.

[42] Q. Zhang, H. Tao, Y. Lin et al., "A superoxide dismutase/ catalase mimetic nanomedicine for targeted therapy of inflammatory bowel disease," Biomaterials, vol. 105, pp. 206221, 2016.

[43] S. H. Jalalian, S. M. Taghdisi, N. S. Hamedani et al., "Epirubicin loaded super paramagnetic iron oxide nanoparticleaptamer bioconjugate for combined colon cancer therapy and imaging in vivo," European Journal of Pharmaceutical Sciences, vol. 50, no. 2, pp. 191-197, 2013.

[44] T. Kanda, K. Ishii, H. Kawaguchi, K. Kitajima, and D. Takenaka, "High signal intensity in the dentate nucleus and globus pallidus on unenhanced T1-weighted MR images: relationship with increasing cumulative dose of a gadoliniumbased contrast material," Radiology, vol. 270, no. 3, pp. 834841, 2014.

[45] V. Gulani, F. Calamante, F. G. Shellock, E. Kanal, and S. B. Reeder, "Gadolinium deposition in the brain: summary of evidence and recommendations," The Lancet Neurology, vol. 16, no. 7, pp. 564-570, 2017.

[46] D. G. Reid and P. S. Murphy, "Fluorine magnetic resonance in vivo: a powerful tool in the study of drug distribution and metabolism," Drug Discovery Today, vol. 13, no. 11-12, pp. 473-480, 2008. 